\title{
Diagnostic Performance and Image Quality of 20- second Breath-hold Total-body PET/CT Acquisition in Stage la Pulmonary Adenocarcinoma
}

\section{Yanhua Duan}

shandong provincial Qianfoshan Hospital

Minjie Zhao

Shandong Provincial Qianfoshan Hospital

Keyu Zan

Shandong provincial Qianfoshan Hospital

Ying Wang

Central Research Institute, United Imaging Healthcare

Xiao Cui

Shandong Provincial Qianfoshan Hospital

Kun Li

Shandong provincial Qianfoshan Hospital

Leiying Chai

Shandong Provincial Qianfoshan Hospital

Wen Liu

Shandong Provincial Qianfoshan Hospital

Min Ge

Shandong Provincial Qianfoshan Hospital

\section{Ximing Wang}

Shandong Provincial hospital

Li Chen

Shandong Provincial Hospital

Zhaoping Cheng ( $\sim$ dyh8212@126.com )

Shandong provincial Qianfoshan Hospital

\section{Research Article}

Keywords: Diagnostic performance, image quality, Breath-hold PET/CT, Total-body PET, Pulmonary adenocarcinoma, Stage IA

Posted Date: July 7th, 2021 
DOl: https://doi.org/10.21203/rs.3.rs-670774/v1

License: (c) (1) This work is licensed under a Creative Commons Attribution 4.0 International License. Read Full License 


\section{Abstract}

\section{Purpose}

The study is to evaluate the diagnostic performance and image quality of a 20-second breath-hold (BH) ${ }^{18}$ F-FDG total-body PET acquisition compared with a free-breathing (FB) PET for stage IA pulmonary adenocarcinoma.

Materials and Methods

Forty-seven patients with confirmed stage IA pulmonary adenocarcinoma were enrolled. All patients underwent total-body ${ }^{18} \mathrm{~F}$-FDG PET/CT and the acquisition time was $300 \mathrm{~s}$, followed by a 20-s BH PET. A 20-s FB PET was extracted from the 300-s PET. The size and volume of lesions were measured on BHCT images. The SUV max $_{\text {, }}$ tumor-to-background ratio (TBR), metabolic tumor volume (MTV), \% $\Delta S U V_{\text {max }}$ and $\% \triangle T B R$ of the lesions were measured and recorded. The lesions were further divided by distance from pleura, lesion size, and morphological characteristic for subgroup analysis.

Results

In the cohort and subgroup analysis, the SUV $\mathrm{max}_{\text {ax }}$ and TBR were significantly increased with 20-BH PET compared with 300-FB PET and 20-FB PET (all p $₫ 0.05$ ). And the $\% \Delta S U V_{\max }$ and $\% \Delta T B R$ in D1 groups ( $\leq 10 \mathrm{~mm}$ in distance) higher than those in $\mathrm{D} 2$ and $\mathrm{D} 3$ groups $(\nabla 10 \mathrm{~mm})$. The diagnostic performance of $\mathrm{BH}$ PET was significantly higher than that of FB PET (all pه0.001). The Bland-Altman plot for agreement on lesion's volume between BH PET and CT showed good agreement than FB PET.

Conclusion

The 20-s BH PET acquisition is more sensitive to quantitative and qualitative analysis for stage IA pulmonary adenocarcinoma. 20-s BH PET/CT acquisition reduces the blurring effect of respiratory motion especially for subpleural nodules ( $\leq 10 \mathrm{~mm}$ in distance).

\section{Introduction}

Five-year survival rate for Non-small cell lung cancer (NSCLC) varied from $92 \%-68 \%$ in patients with stage IA-IB to $0 \%-10 \%$ in patients with stage IVA-IVB [1]. Mortality of NSCLC can be reduced by identifying lung cancer at an early stage when treatment can be more effective. So, it's important to diagnose the pulmonary nodules at an early stage. With the development of high-resolution computed tomography (HRCT), the detection rate of small lung lesions has been improved, especially in stage IA lung adenocarcinoma (smaller than $3 \mathrm{~cm}$ in diameter without lymphatic and distant metastasis) [2-3]. However, the fluorine-18-fluorodeoxyglucose ${ }^{18} \mathrm{~F}$-FDG PET/CT, combining anatomic data with functional and metabolic information, has been confirmed to provide a higher sensitivity, specificity and accuracy 
than PET or CT alone for evaluating the lung nodules, especially for solid pulmonary nodules (SPNs) and mixed ground-glass opacity nodules (mGGNs) [4-7].

Whole-body PET imaging requires an acquisition of $\sim 3$ min per bed position and respiratory motion artifacts have a significant effect on the image quality, distortions of lesion size, shape, location and quantification of radiotracer uptake $[8,9]$. Amplitude-based respiratory gating technique has been used to reduce the respiratory motion blurring. However, it's time consuming because of extra breathing training and repeated examination due to poor synchronization of breathing $[8,10]$, and sometimes it has potential radiation hazard to technicians.

Deep-inspiration breath-hold PET/CT technique has been demonstrated to minimize respiratory motion artifacts and PET/CT image misregistration [10, 11], allowing higher standard uptake value (SUV) for pulmonary nodules, and has been proven with a higher diagnostic accuracy for malignant lesions than the standard FB PET [10]. However,previous studies were performed on the PET/CT scanners with a standard axial field of view (AFOV) of $\sim 30 \mathrm{~cm}$ and limited system sensitivity. Thus, the acquired counts in a single breath-hold cycle on such scanners were not adequate, and the image quality and confidence for diagnosis were reduced. The total-body PET/CT system (uEXPLORER, United Imaging Healthcare, Shanghai, China), equipped with a 194-cm-long AFOV, can offer significant improved sensitivity over previous scanners $[12,13]$. This sensitivity gain can be utilized to shorten the acquisition time while maintaining comparable image quality and lesion detectability in oncological studies [14-16]. Thus, $\mathrm{BH}$ PET with a total-body PET/CT system is of great potential in lung cancer imaging.

Previous studies reported the evaluation of the blurring effect of the respiratory motion in comparison with the BH PET/CT imaging. To the best of our knowledge, there are few studies that comprehensively investigate the factors of respiratory motion on pulmonary nodules that may influence the diagnostic efficiency and image quality of PET acquisition. This study aims to evaluate the diagnostic performance and image quality of a single 20-s BH PET acquisition compared with the FB PET imaging, and investigates various factors contributed to the respiratory motion including lesion morphological characteristics, size and distance from pleura.

\section{Materials And Methods}

\section{Patients}

This prospective study was approved by the institutional review board of The First Affiliated Hospital of Shandong First Medical University, and written informed consent was obtained from all the patients. The study was conducted from May 2020 to December 2020, and100 consecutive patients with solitary solid pulmonary nodules and mGGNs detected by CT at local hospitals for suspected malignancy were included in the study. All patients referred to our department underwent total-body ${ }^{18} \mathrm{~F}-\mathrm{FDG} \mathrm{PET} / \mathrm{CT}$ examinations for diagnosis or cancer staging. 
Figure 1 shows the flow chart of patient recruitment. The inclusion criteria were as follows: (1) solitary solid nodules or mixed ground-glass nodules (mGGNs) with a diameter of less than $30 \mathrm{~mm}$ (measured in the CT images using the lung window) without associated atelectasis and pneumonia; (2) patients were not treated with radiotherapy, chemotherapy or other specific oncologic treatments before ${ }^{18} \mathrm{~F}-\mathrm{FDG}$ $\mathrm{PET} / \mathrm{CT}$ examination; (3) patients could hold their breath for $20 \mathrm{~s}$ or longer during a training performed by a technician before the examination.

After 20-BH PET, the patients with confirmed adenocarcinoma by surgical or image-guided biopsy pathology were enrolled in this study, and the excluded patients included patients with squamous carcinoma $(n=6)$, adenosquamous carcinoma $(n=2)$, neuroendocrine carcinoma $(n=3)$, benign lesions $(n=7)$, adenocarcinoma in situ (AIS, $n=5)$, atypical adenomatous hyperplasia (AAH, $n=3)$, small cell lung cancer $(n=2)$, metastases $(n=4)$, and unknown lesions $(n=21)$. Patients were also excluded if multiple secondary lung nodules were identified in 300-FB PET images.

Forty-seven patients (female/male: 22/25), confirmed with solitary pulmonary adenocarcinoma by surgical resection ( $n=20)$ or image-guided biopsy pathology $(n=27)$, were finally enrolled in this study. The demographic and clinical characteristics of the enrolled patients are listed in Table 1.

\section{PET/CT examination}

All patients received a weight-based injection of ${ }^{18} \mathrm{~F}-\mathrm{FDG}(2.96 \mathrm{MBq} / \mathrm{kg})$ after fasting for at least 6 hours, and blood glucose levels (measured with the finger-stick method) were controlled less than $170 \mathrm{mg} / \mathrm{dL}$. PET/CT examination was performed approximately $60 \mathrm{~min}$ after the administration $(67.94 \pm 6.85 \mathrm{~min})$.

PET/CT examinations were performed on a total-body PET/CT system. A low dose whole-body spiral CT with free breathing protocol was acquired for attenuation correction and anatomic reference. The $x$-ray tube voltage was set to 80 and $100 \mathrm{kV}$ for patients with body mass index (BMI) less than $25 \mathrm{~kg} / \mathrm{m}^{2}$ and greater than $25 \mathrm{~kg} / \mathrm{m}^{2}$, respectively. A referenced tube current of $100 \mathrm{~mA}$ was used with automatic tube current modulation technique. The whole body free-breath CT scan (referred as FBCT) was acquired with $3.0 \mathrm{~mm}$ slices $(160 \times 0.5 \mathrm{~mm})$, matrix size of $512 \times 512$, a pitch of 0.9625 and a rotation time of $0.5 \mathrm{~s}$. A 300-s free-breath PET acquisition (referred as 300-FB PET) was performed immediately after the CT scan, with no change in patient position. A 20-s free-breath PET (referred as 20-FB PET) was extracted from the last 20-s of the list-mode 300-FB PET raw data.

A diagnostic thoracic CT (referred as BHCT) was performed after the 300-s PET acquisition, during which the patient was instructed to hold the breath at maximum inspiration. Then, the patient underwent a 20-s BH PET (referred as 20-BH PET) acquisition with breath holding.

All PET images were reconstructed using the ordered subset expectation maximization (OSEM) algorithm with the following parameters: TOF and PSF modeling, 3 iterations and 20 subsets, matrix of $192 \times 192$, slice thickness of $2.886 \mathrm{~mm}$, FOV $600 \mathrm{~mm}$ with a full width at half maximum of $3 \mathrm{~mm}$ in Gaussian postfilter, and all the necessary corrections, such as attenuation and scatter correction. 


\section{Image analysis}

All PET/CT images were anonymous and transferred to a commercial medical image processing workstation (uWS-MI, United Imaging Healthcare, Shanghai, China) for analysis.

\section{Quantitative analysis}

The quantitative analysis was assessed by a nuclear radiologist with 5-year experience of oncological radiology (including 1 year of PET/CT). For each patient, three 3D spherical volume-of-interests (VOIs) with a diameter of $1 \mathrm{~cm}$ were drawn on a homogeneous area of the descending aorta away from the edge and calcification. The SUV mean values of the VOIs were recorded and averaged to ensure data consistency. Another VOI was placed in the target pulmonary nodule with a threshold of $50 \%$ of the $S_{S U} V_{\max }$ value within the contour margin that can automated define the boundaries of the nodules. The

SUV $_{\text {max }}$ and metabolic tumor volume (MTV) of the pulmonary nodules were measured. For each nodule, tumor-to-background ratio (TBR) was calculated by dividing the nodule SUV max $_{\text {ax }}$ by the descending aorta SUV $V_{\text {mean }}$.

Subgroup analyses were performed to investigate the factors of the respiratory motion. The lesions were firstly divided into solid pulmonary nodule group (G1) and mixed ground-glass nodules (mGGNs) group (G2) by the morphological characteristics [3]. Lesions were further divided into T1a lesions ( $\leq 10 \mathrm{~mm}$ in longest axis), T1 b lesions (10-20 mm in longest axis) and T1c lesions (20-30 mm in longest axis) by lesion size. In another subgroup analysis, lesions were divided into D1 ( $\leq 10 \mathrm{~mm})$, D2 $(10-20 \mathrm{~mm})$ and

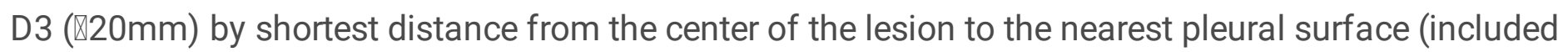
diaphragm) [17].

The percentage difference between the 300 -s PET and BH PET (referred as $\% \Delta S \mathrm{SU}_{\max }$ and $\% \Delta \mathrm{TBR}$ ) were obtained as the following equations [18]:

$\% \Delta S U V_{\max }=\left(S_{\max B H^{-}} \mathrm{SUV}_{\max 300-\mathrm{FB}}\right) / \mathrm{SUV}_{\max 300-\mathrm{FB}} \times 100$

$\% \Delta T B R=\left(T B R_{B H}-T B R_{300-F B}\right) / T B R_{300-F B} \times 100$.

The size (longest axis), volume and distance from pleura of each lesion were measured in the BHCT images.

\section{Visual analysis}

All PET/CT images were visually analyzed in a randomized and unpaired order. Blinded to the patients' characteristics and PET acquisitions, two nuclear radiologists with 10-year experience in interpreting PET images independently identified the nodules by visually checking the suspected lesion uptake (FDG positive findings were defined as lesion uptake above background, and FDG negative findings were 
defined as lesion uptake at/below-background)[19]. The reference organ for background was the descending aorta.

Another two radiologists with 5-year experience in interpreting CT images independently diagnose the nodules by BHCT images; consensus agreement was determined by an escalated radiologist if disagreement existed.

\section{Statistical Analysis}

Statistical analysis was performed using IBM SPSS software package (version 20.0, IBM Corporation, New York, USA) and MedCalc software (version 13.0.2, MedCalc for Windows, Mariakerke, Belgium).

Continuous data were expressed as a mean \pm standard deviation and categorical data were displayed as absolute frequencies or proportions. Difference was expressed as relative changes. A p value $<0.05$ was considered to indicate a significant difference.

Patients' characteristics (e.g. age, body weight, height, BMI and Blood glucose level) and FDG injection parameters were compared between subgroups using independent $\mathrm{t}$ test or Kruskal-Wallis $\mathrm{H}$ test. Quantitative parameters ( $\mathrm{SUV}_{\text {max }}, \mathrm{MTV}, \mathrm{TBR}$ and $\% \Delta$ ) were compared among 300-FB PET, 20-FB PET and 20-BH PET acquisitions using Wilcoxon rank-sum test. The correlations between quantitative parameters, lesion ingredient, size, and distance to pleura were analyzed by Spearman's rank correlation coefficient. Qualitative parameters were compared by the Chi-square test or Fisher exact test. The agreement between MTV of the different PET acquisitions and volume of BHCT images was further examined using a BlandAltman plot.

\section{Results}

\section{Patient characteristics}

A total number of 47 patients with stage IA pulmonary adenocarcinoma were included in the study; and patients' characteristics are summarized in Table 1.

Nodules were classified as $\mathrm{G} 1(\mathrm{n}=26)$ and $\mathrm{G} 2(\mathrm{n}=21)$ by morphological characteristics of nodules. Lesions were further divided into T1a $(n=9), T 1 b(n=17), T 1 c(n=21)$ by lesion size and D1 $(n=12), D 2$ $(n=15)$ and $D 3(n=20)$ by distance from pleura for subgroup analysis. There were no significant

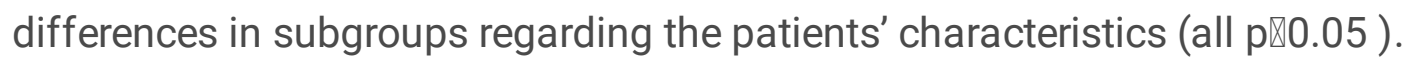

\section{Nodule analysis: entire cohort}

The 20-BH PET images can improve the lesion conspicuousness compared with the 300-FB PET and 20FB PET images (as shown in Fig. 2). The SUV $\mathrm{max}_{\max }$ and TBR of all the 47 lesions of the 20-BH PET were significantly higher than those of the 300-FB PET (both $p<0.01$ ) and 20-FB PET ( $p=0.034$ and $p<0.001$, respectively), while the MTV was significantly reduced (both $p<0.001$ ), as shown in Fig. 3 . There were no 
significant differences between the 300-FB PET and 20-FB PET regarding the lesion SUV $\mathrm{max}_{\max }$ and TBR $(\mathrm{p}=$ 0.180 and $p=0.063$, respectively). There was no significant difference between the 300-FB PET and 20BH PET regarding the background SUV mean $(p=0.297)$, while the background SUV mean of 20-FB PET was significantly higher than that of 300-FB PET and 20-BH PET (both $p<0.001$ ).

\section{Nodule analysis: subgroups}

In the subgroup analysis, the SUV max $_{1}$ TBR and MTV of both G1 and G2 groups in 20-BH PET were significantly different compared with those in 300-FB PET and 20-FB PET (all pष0.05). And there were no significant difference between 300-FB PET and 20-FB PET (all p囚0.05). Lesion SUV max, TBR, MTV as well as background SUV $V_{\text {mean }}$ values of G1 and G2 groups are summarized in Fig. 4.

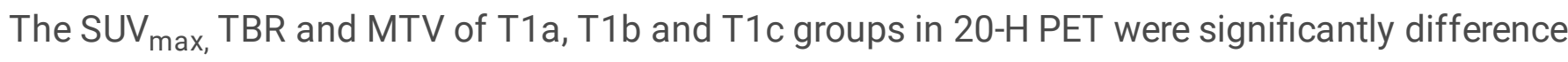

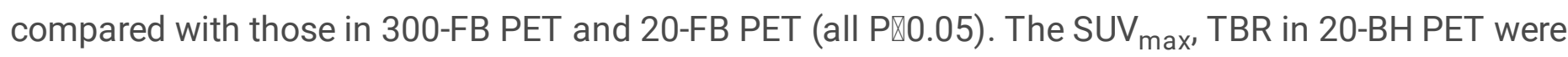
significantly higher than those in 300-FB PET and 20-FB PET regardless of the lesion size (Fig. 5).

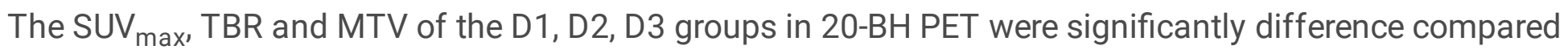

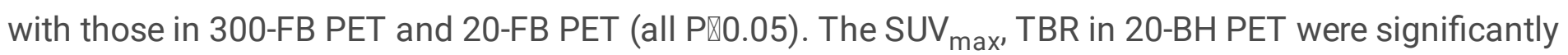
higher than those in 300-FB PET and 20-FB PET regardless of the distance from lesions to pleura (Fig. 6).

There were no significant differences between $\mathrm{G} 1$ and $\mathrm{G} 2$ groups regarding the $\% \Delta S U V_{\max }$ and $\% \Delta T B R$ $(p=0.480$ and $p=0.881$, respectively). There were no significant differences between different size regarding the $\% \Delta S U V_{\text {max }}$ and $\% \triangle T B R\left(p=0.428\right.$ and $p=0.488$, respectively). However, the $\% \Delta S U V_{\max }$ and $\% \triangle T B R$ in T1a groups were higher than those in T1b and T1c groups.

There was significant difference between D1, D2 and D3 groups regarding the $\% \Delta \operatorname{SUV}_{\max }(p=0.003)$, and the $\% \Delta S U V_{\max }$ and \% $\triangle T B R$ in D1 group were higher than those in D2 and D3 groups. The effect of inspiration on $\% \Delta S U V_{\max }$ was significantly high for nodules adjacent to pleura ( $\leq 10 \mathrm{~mm}$ in distance). Lesion \% $\triangle$ SUV $_{\max }$ and TBR values of subgroups are summarized in Table 2.

\section{Nodule analysis: volume}

For all the 47 lesions, the mean lesion volume with BHCT was $2101.73 \pm 1531.36 \mathrm{~mm}^{3}$. A good agreement was shown between the MTV of the 47 lesions in different PET acquisitions and volume of BHCT (as shown in Fig. 7). The best agreement was found between the MTV in 20-BH PET and volume of BHCT, indicating an accurate measurement of the nodules, as shown in Fig. 8.

\section{Diagnostic Performance}

Of the 47 adenocarcinoma nodules, 33 malignant nodules and 14 benign nodules were diagnosed by BHCT, 45 FDG-positive nodules and 2 FDG-negative nodules were diagnosed by 20-BH PET/CT, 42 FDG- 
positive nodules and 5 FDG-negative nodules were diagnosed by 300-PET/CT, 40 FDG-positive nodules and 7 FDG-negative nodules were diagnosed by 20-FB PET/CT.

The diagnostic performance of BHCT, 300-FB PET/CT, 20-FB PET/CT and 20-BH PET/CT were 70.21\%, $89.36 \%, 85.11 \%$ and $95.74 \%$, respectively, and the sensitivity of the fused images acquired by the PET/CT

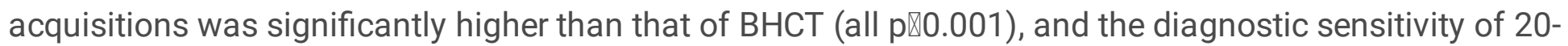
$s$ BH PET/CT was significantly higher than that of 300-s PET/CT (p『0.001) and 20-FB PET/CT $(p=$ $0.001)$.

\section{Discussion}

This study was designed to assess the diagnostic efficiency and image quality of a 20-BH PET acquisition compared with a 20- and 300-FB PET for stage IA pulmonary adenocarcinoma. The 20-BH PET resulted in reduced breathing-induced artifacts on PET images of the lung. The main findings are as follows: (a) 20-BH PET acquisition provided an increased nodule SUV max $_{\text {ax }}$ and TBR across the cohort, regardless of its morphological characteristic, size and distance from the pleura; (b) 20-BH PET acquisition provided very closely MTV of nodules compared with BHCT; (c) there was an inverse correlation between nodule distances from pleura and $\% \Delta S U V_{\text {max }}$; and (d) when 20-BH PET was performed, the diagnostic performance of fused PET/CT in stage IA pulmonary adenocarcinoma was higher than that of BHCT, and 20-BH PET/CT provided a higher accuracy than 20- and 300-FB PET/CT.

The accuracy of lung nodule characterization with PET is affected by several physical factors of the PET scanner, such as system resolution and spatial resolution, particularly when the delineation of the target lesion is affected by the respiratory motion. The total-body PET scanner used in the study implemented the pixilated LYSO crystals, with a size of $2.76 \times 2.76 \times 18.1 \mathrm{~mm}^{3}$, yielding an excellent spatial resolution of $~ 3 \mathrm{~mm}$ according to the NEMA NU 2-2018 [12]. Moreover, the AFOV of this total-body PET scanner is up to $194 \mathrm{~cm}$ which can effectively detected the photons emitted from the patients. The elongated AFOV leads to an approximately 40 -fold increase in the effective sensitivity according to the simulations [13]. The signal-to-noise ratio (SNR), a measure representing the PET image quality, is proportional to the square root of the product of the scanner sensitivity, injected activity, and the total acquisition duration. As such, the improved sensitivity can improve SNR, or allow for short acquisition duration while maintaining the image quality. Therefore, a 20-s BH PET acquisition is feasible with the total-body $\mathrm{PET} / \mathrm{CT}$ scanner while maintain a good image quality.

Due to the limited sensitivity of the PET scanners with a standard AFOV of $\sim 30 \mathrm{~cm}$, efforts have been made to accurately characterize the lung nodules. First attempt is to increase the acquisition duration. In a previous study on the feasibility of the BH PET acquisition in lung cancer, patients were instructed to hold their breath as long as possible to detect more counts and improve the image quality. Patients whose breath lasted fewer than 29 seconds were excluded and the study demonstrated that more than $81.9 \%$ (95/116) of the enrolled patients can successfully provide a breath-hold PET acquisition ranging from 30-143 seconds [19]. If the acquisition duration was further reduced, we believed that the proposed 
PET breath-hold acquisition can make a high probability in completing the scan. Our data suggest that a single 20-s BH acquisition is feasible for most patients.

Advantage of the proposed BH PET protocol included the short time for the additional BH PET acquisition, a high probability of technical success in completing the study in cooperative patients, simple post-processing and no additional radiation exposure.

In another study, three consecutive BH PET acquisitions were performed instead of a single $\mathrm{BH}$ acquisition. Moreover, SUV $_{\text {peak }}$ was used to provide a more accurate characterization of lung nodules than SUV max to minimize the effect of the statistical noise due to the low counts [10]. The repeated acquisitions will increase the total acquisition duration and require post-processing if applied in the clinical practice. All the above-mentioned limitations can be overcome by the total-body PET/CT scanner, where enough counts can be collected within 20 seconds.

Previous studies have reported an improved accuracy of the lesion uptake and metabolic volume when an external device was utilized for respiratory gating [19-21]. Respiratory motion has been successfully corrected for lung nodules either benign or malignant. PET reconstruction is performed using PET raw data divided into several slot bins between each gate signal. In order to maintain a constant image quality, the acquisition duration should be multiplied by the number of the slot bins. Therefore, the PET acquisition duration has been increased and patient comfort and throughput has been decreased. Moreover, the method requires additional time for patient setup and introduces additional radiation exposure to the operators. Alternatively, a data-driven respiratory gating (DDG) method has been introduced $[22,23]$. It represents an efficient breakthrough in terms of respiratory motion correction without additional work for patient setup. DDG method can increase the SUV max $_{\text {of }}$ the lesion and decrease the threshold-defined lesion volume. Moreover, it can even provide performance superior to that of the external device-based method. Likewise, DDG method also requires multiple acquisition duration to detect enough counts to yield a comparable image quality.

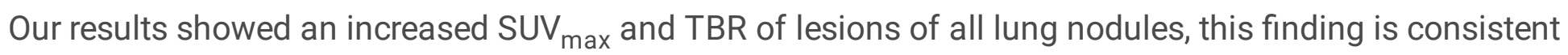
with previous studies $[10,19,24]$. Our data also analyzed the effects of the characteristics of nodules on the SUV max $_{\text {and TBR, and the SUV }}$ max and TBR increased regardless of its morphological characteristic, size and distance from the pleura. However, there was an inverse correlation between distance from the pleura and $\% \Delta S U V_{\max }$ and $\% \Delta T B R$, for subpleural nodules ( $\leq 10 \mathrm{~mm}$ in distance), the $\% \Delta S U V_{\text {max }}$ and

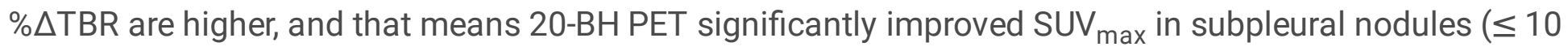
$\mathrm{mm}$ in distance). In addition, the increasing amplitude of SUV $\mathrm{max}_{\text {and }}$ aBR increases for nodules $\leq 10$ $\mathrm{mm}$ is greater than the increase for nodules $₫ 10 \mathrm{~mm}$. This should enable $20-\mathrm{BH}$ PET to be used in the assessment of subpleural and smaller nodules.

Our data showed that metabolic volumes of lung nodules are decreases on average by applying breathhold technique. Figure 4 shows subpleural lesion was blurred and expanded inaccurately due to the influence of breathing on 300- and 20-FB PET images, but on 20-BH PET images, the quantitative MTV as 
well as the boundary of the lesion were effectively corrected. Consistent with previous study [23],these differences are likely due to the subpleural nodules, which are close to the pleura and heavily affected by respiration.

The effect of breath-hold technique on nodule evaluation and diagnostic performance was assessed using semi-quantitative and visual criteria. Our results showed that using 20-BH PET/CT achieved a higher diagnostic efficiency than CT alone or 300- and 20-FB PET/CT, and suggested that the 20-BH $\mathrm{PET} / \mathrm{CT}$ fusion may present advancement in stage IA pulmonary adenocarcinoma applications.

\section{Limitations:}

There are several limitations in our study. Firstly, only patients with stage IA pulmonary adenocarcinoma was enrolled, which kept the homology of all cases; in the further study, different pathologic types of lung nodules will be enrolled to evaluate the differential diagnosis value of 20-BH PET/CT on lung nodules. A second limitation is the small sample size, and it is necessary to perform a large scale study to improve reliability and validity of 20-BH PET/CT. Thirdly, holding breath for 20-s is not feasible for all patients especially for patients with pulmonary dysfunction, motion correction technology will be used to reduce motion artifacts in cooperative patients in further studies.

\section{Conclusion}

The use of 20-s breath-hold PET/CT results in a significant increase in metabolic activity and tumor-tobackground ratio of nodules in comparison with conventional FB PET/CT. The diagnostic accuracy of 20BH PET/CT for stage IA pulmonary adenocarcinoma is improved; it appears to provide a more accurate measurement on the metabolic tumor volume of the nodules. Moreover, the 20-s breath-hold PET/CT acquisition can reduce the blurring effect of respiratory motion especially for nodules adjacent to pleura ( $\leq 10 \mathrm{~mm}$ in distance).

\section{Declarations}

\section{Funding}

This study is supported by Academic promotion programme of Shandong First Medical University (2019LJ005).

\section{Conflicts of interest/Competing interests}

Y W is a full-time employee of United Imaging Healthcare, China. All the other authors have no conflicts of interest to report.

\section{Availability of data and material}


Data are available on request to the corresponding author.

\section{Code availability}

Not applicable.

\section{Authors' contributions}

Study conception and design: YH D, XM W, L C, ZP C.

Material preparation and data collection: YH D, MJ Z, KY Z, X C, K L, M G.

Data analysis: YH D, Y W, MJ Z, KY Z, X C, W L.

Drafting of the manuscript: YHD, L C, Y W.

All authors commented on previous versions of the manuscript and read and approved the final manuscript.

\section{Ethics approval}

All procedures performed in studies involving human participants were in accordance with the ethical standards of the institutional and/or national research committee and with the 1964 Helsinki declaration and its later amendments or comparable ethical standards.

\section{Consent to participate}

All the enrolled patients have signed the informed consent prior to the examination.

\section{Consent for publication}

Patients signed informed consent regarding publishing their data and photographs for scientific use.

\section{References}

1. Goldstraw P, Chansky K, Crowley J, et al. The IASLC lung cancer staging project: proposals for revision of the TNM stage groupings in the forthcoming (eighth) edition of the TNM classification for lung cancer. J Thorac Oncol. 2016;11(1):39-51.

2. Truong MT, Ko JP, Rossi SE, et al. Update in the evaluation of the solitary pulmonary nodule. Radiographics. 2014 Oct;34(6):1658-79. 
3. National Lung Screening Trial Research Team. Aberle DR, Adams AM, et al. Reduced lung-cancer mortality with low-dose computed tomographic screening. N Engl J Med. 2011;365(5):395-409.

4. Gould MK, Maclean CC, Kuschner WG, et al. Accuracy of positron emission tomography for diagnosis of pulmonary nodules and mass lesions: a meta-analysis. JAMA. 2001;285(7):914-24.

5. Kagna O, Solomonov A, Keidar Z, et al. The value of FDG-PET/CT in assessing single pulmonary nodules in patients at high risk of lung cancer. Eur J Nucl Med Mol Imaging. 2009;36(6):997-1004.

6. Cronin P, Dwamena BA, Kelly AM, et al. Solitary pulmonary nodules: meta-analytic comparison of cross-sectional imaging modalities for diagnosis of malignancy. Radiology. 2008;246(3):772-82.

7. Kandathil A, Kay FU, Butt YM, et al. Role of FDG PET/CT in the Eighth Edition of TNM Staging of Non-Small Cell Lung Cancer. Radiographics. 2018;38(7):2134-49.

8. Teo BK, Saboury B, Munbodh R, et al. The effect of breathing irregularities on quantitative accuracy of respiratory gated PET/CT. Med Phys. 2012;39(12):7390-7.

9. Nehmeh SA, Erdi YE, Pan T, et al. Quantitation of respiratory motion during 4D-PET/CT acquisition. Med Phys. 2004;31(6):1333-8.

10. Balamoutoff N, Serrano B, Hugonnet F, et al. Added Value of a Single Fast 20-second DeepInspiration Breath-hold Acquisition in FDG PET/CT in the Assessment of Lung Nodules. Radiology. 2018;286(1):260-70.

11. Shyn PB, Tatli S, Sainani NI, et al. Minimizing image misregistration during PET/CT-guided percutaneous interventions with monitored breath-hold PET and CT acquisitions. J Vasc Interv Radiol. 2011;22(9):1287-92.

12. Spencer BA, Berg E, Schmall JP, et al. Performance evaluation of the uEXPLORER Total-body PET/CT scanner based on NEMA NU 2-2018 with additional tests to characterize long axial field-of-view PET scanners [published online ahead of print, 2020 Oct 2]. J Nucl Med. 2020; jnumed.120.250597.

13. Cherry SR, Jones T, Karp JS, et al. Total-Body PET: Maximizing Sensitivity to Create New Opportunities for Clinical Research and Patient Care. J Nucl Med. 2018;59(1):3-12.

14. Zhang YQ, Hu PC, Wu RZ, et al. The image quality, lesion detectability, and acquisition time of $18 \mathrm{~F}-$ FDG total-body PET/CT in oncological patients. Eur J Nucl Med Mol Imaging. 2020;47(11):2507-15.

15. Zhao YM, Li YH, Chen T, et al. Image quality and lesion detectability in low-dose pediatric 18F-FDG scans using total-body PET/CT [published online ahead of print, 2021 Mar 18]. Eur J Nucl Med Mol Imaging. 2021;10.1007/s00259-021-05304-4.

16. Hu P, Zhang Y, Yu H, et al. Total-body 18F-FDG PET/CT scan in oncology patients: how fast could it be? [published online ahead of print, 2021 Apr 18]. Eur J Nucl Med Mol Imaging. 2021;10.1007/s00259-021-05357-5.

17. Moreland A, Novogrodsky E, Brody L, et al. Pneumothorax with prolonged chest tube requirement after CT-guided percutaneous lung biopsy: incidence and risk factors. Eur Radiol. 2016;26(10):348391. 
18. Teoh EJ, McGowan DR, Bradley KM, et al. Novel penalised likelihood reconstruction of PET in the assessment of histologically verified small pulmonary nodules. Eur Radiol. 2016;26(2):576-84.

19. Kawano T, Ohtake E, Inoue T. Deep-inspiration breath-hold PET/CT versus free breathing PET/CT and respiratory gating PET for reference: evaluation in 95 patients with lung cancer. Ann Nucl Med. 2011;25(2):109-16.

20. Lupi A, Zaroccolo M, Salgarello M, et al. The effect of 18F-FDG PET/CT respiratory gating on detected metabolic activity in lung lesions. Ann Nucl Med. 2009;23(2):191-6.

21. Nehmeh SA, Erdi YE, Ling CC, et al. Effect of respiratory gating on quantifying PET images of lung cancer. J Nucl Med. 2002;43(7):876-81.

22. Walker MD, Morgan AJ, Bradley KM, et al. Data-Driven Respiratory Gating Outperforms Device-Based Gating for Clinical 18F-FDG PET/CT. J Nucl Med. 2020;61(11):1678-83.

23. Kang SY, Moon BS, Kim HO, et al. The impact of data-driven respiratory gating in clinical F-18 FDG PET/CT: comparison of free breathing and deep-expiration breath-hold CT protocol. Ann Nucl Med. 2021;35(3):328-37.

24. Nehmeh SA, Erdi YE, Meirelles GS, et al. Deep-inspiration breath-hold PET/CT of the thorax. J Nucl Med. 2007;48(1):22-6.

\section{Tables}

Due to technical limitations, table 1 and 2 is only available as a download in the Supplemental Files section.

\section{Figures}




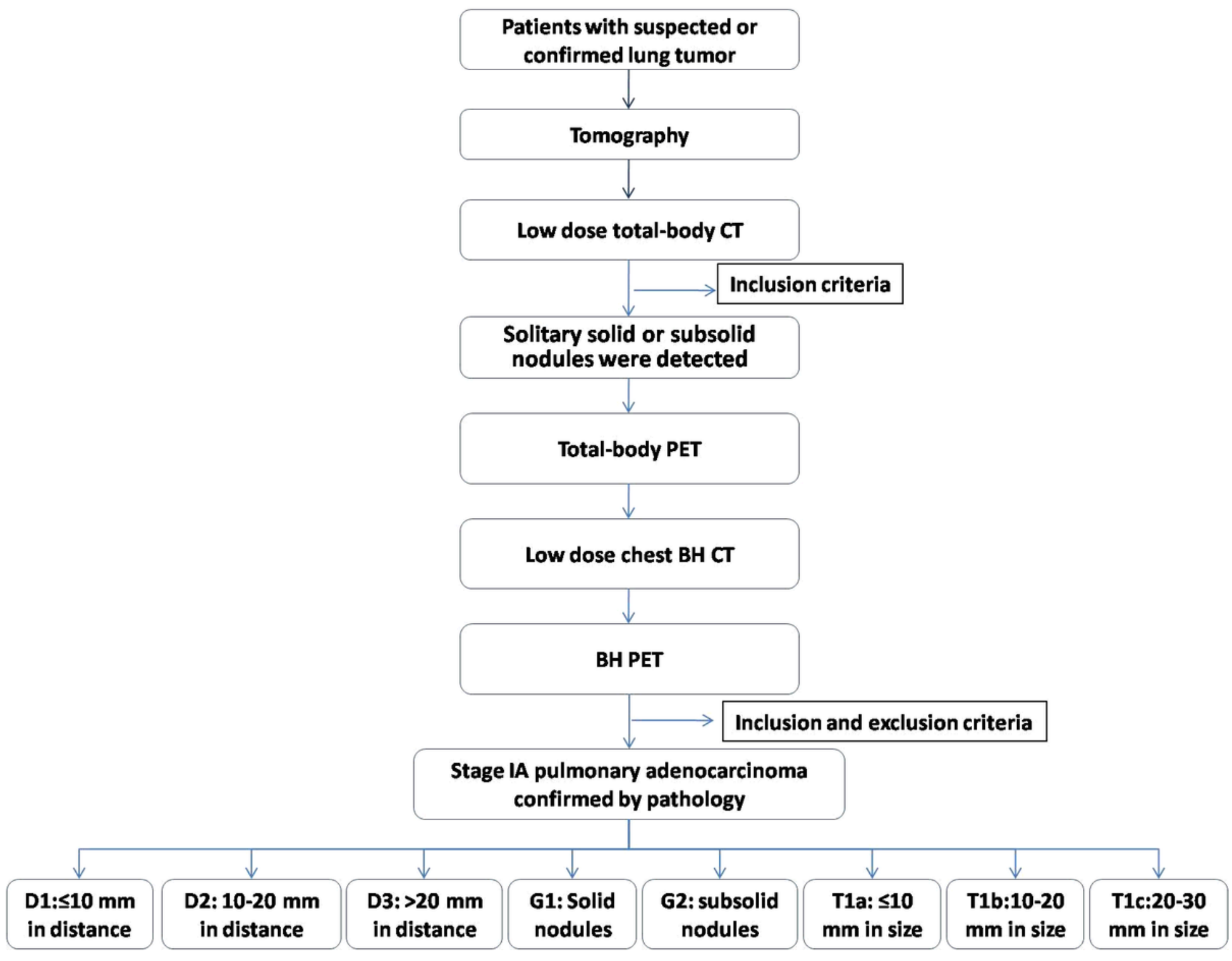

Figure 1

Flow chart of patient recruitment 

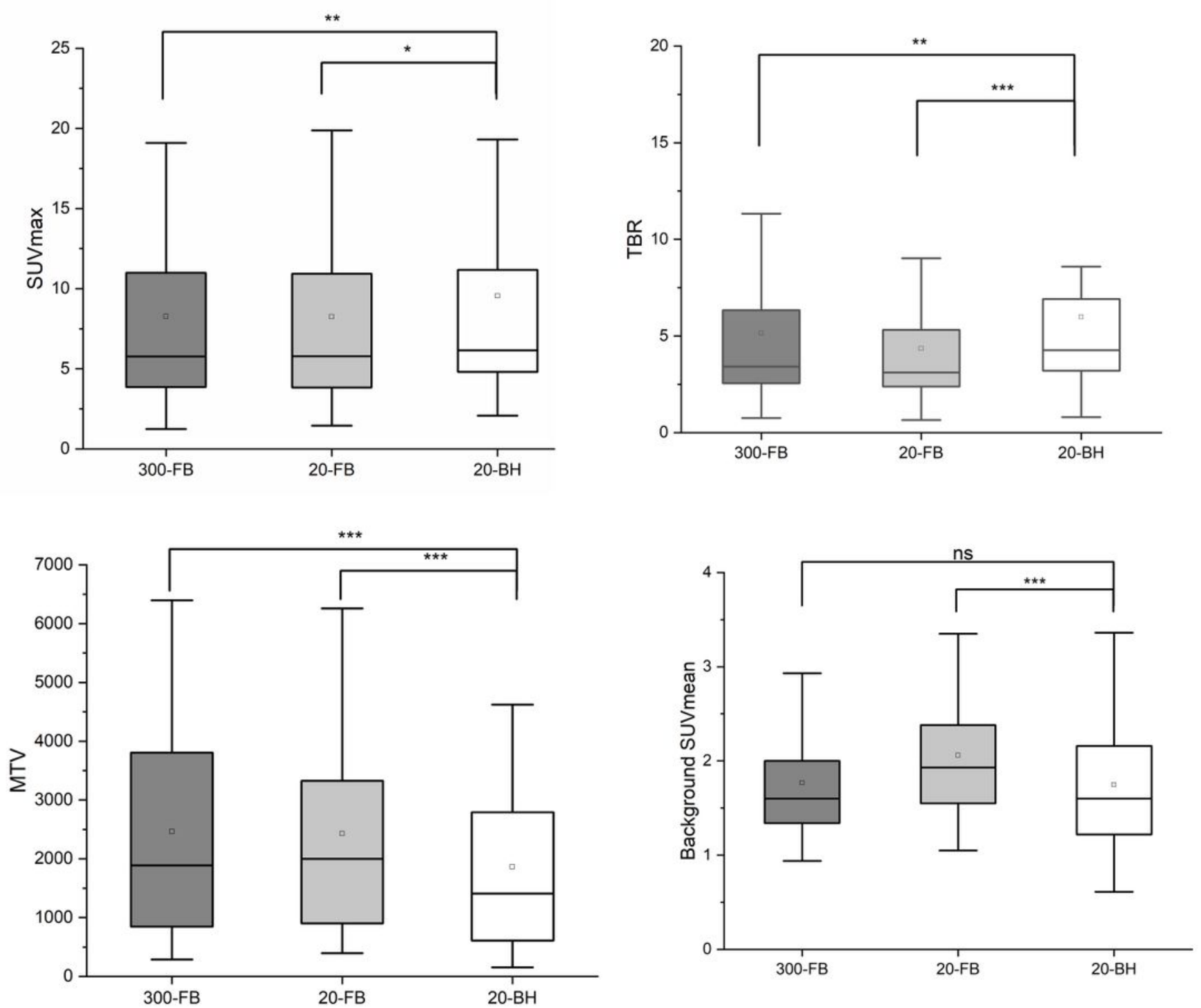

Figure 2

Differences in SUVmax, TBR, MTV and background SUVmean value detected by 300-FB PET (300-FB), 20FB PET (20-FB) and 20-BH PET (20-BH). 


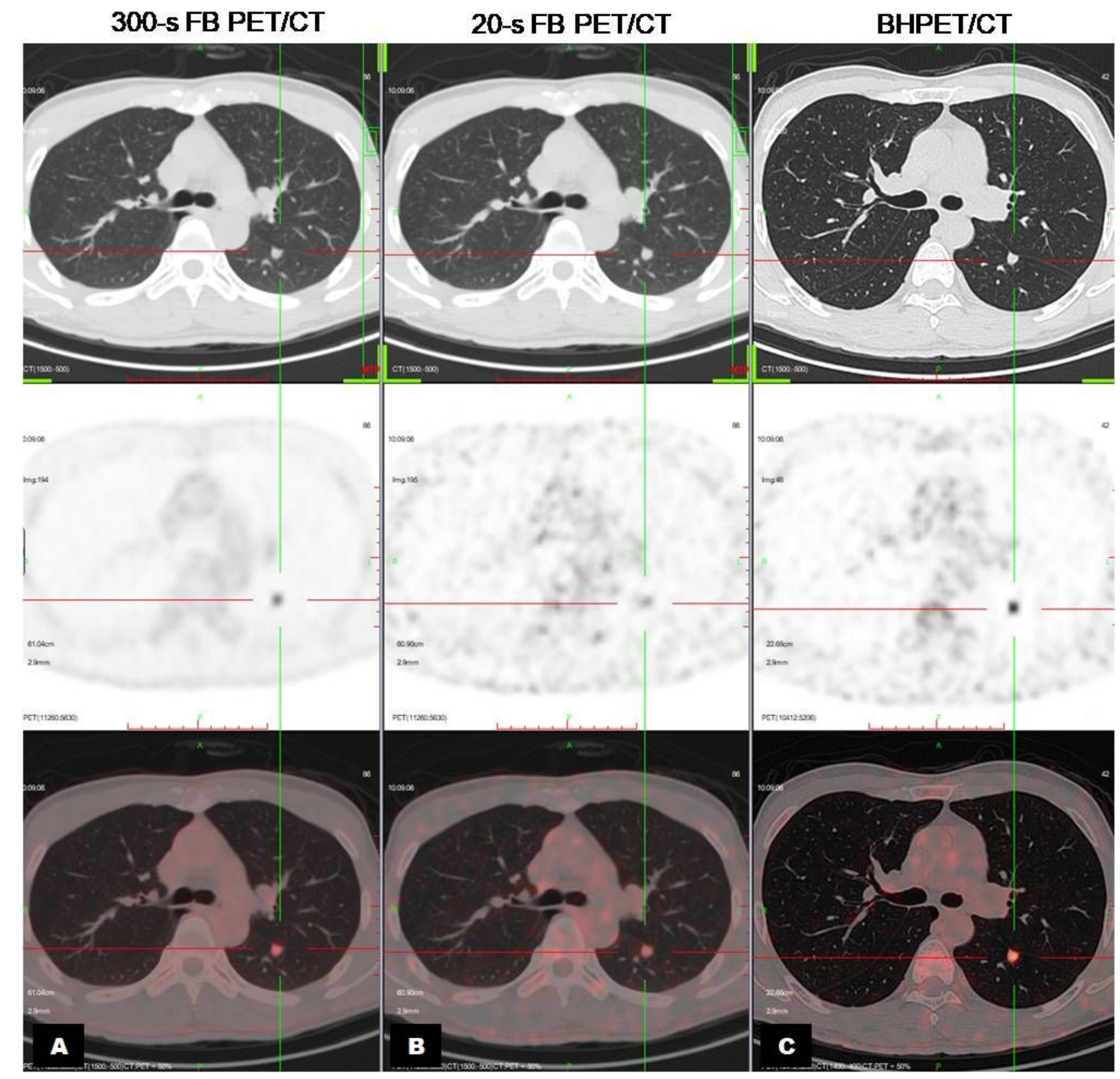

Figure 3

Axial chest CT (top row), PET (middle row) and PET/CT (bottom row) images in 44-year-old man with solitary solid lung nodule (biopsy-proven adenocarcinoma) in left lower lobe. A, 300-s FB PET/CT; B, 20-s FB PET/CT; C, BH PET/CT. Maximum axial diameter of lesion was $9.2 \mathrm{~mm}$. 

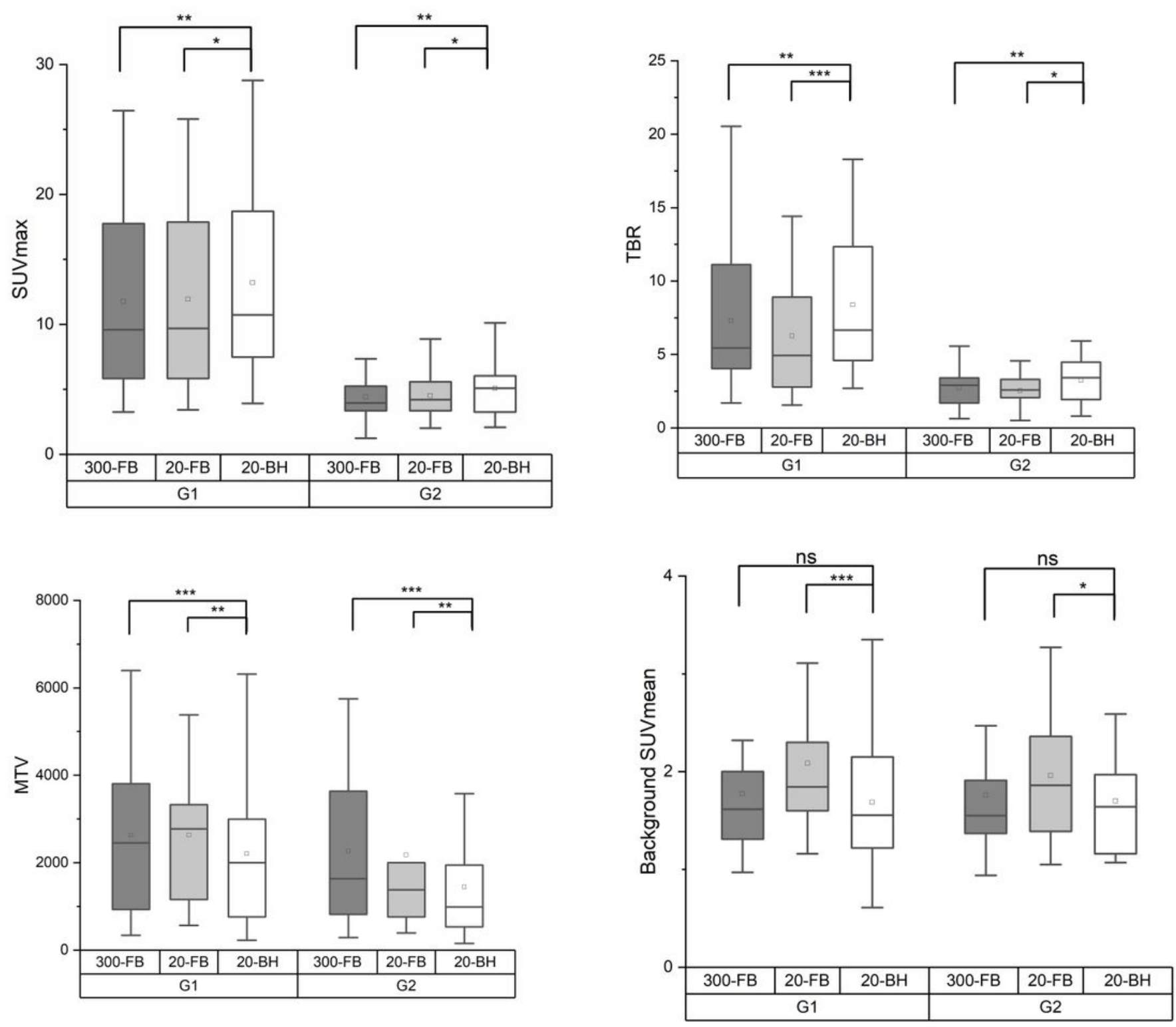

Figure 4

Differences in SUVmax, TBR, MTV and background SUVmean value detected by 300-FB PET (300-FB), 20FB PET (20-FB) and 20-BH PET (20-BH) between G1 and G2 groups.

\section{Figure 5}

Differences in SUVmax, TBR, MTV and background SUVmean value detected by 300-FB PET (300-FB), 20FB PET (20-FB) and 20-BH PET (20-BH) among T1a, T1b and T1c groups. 

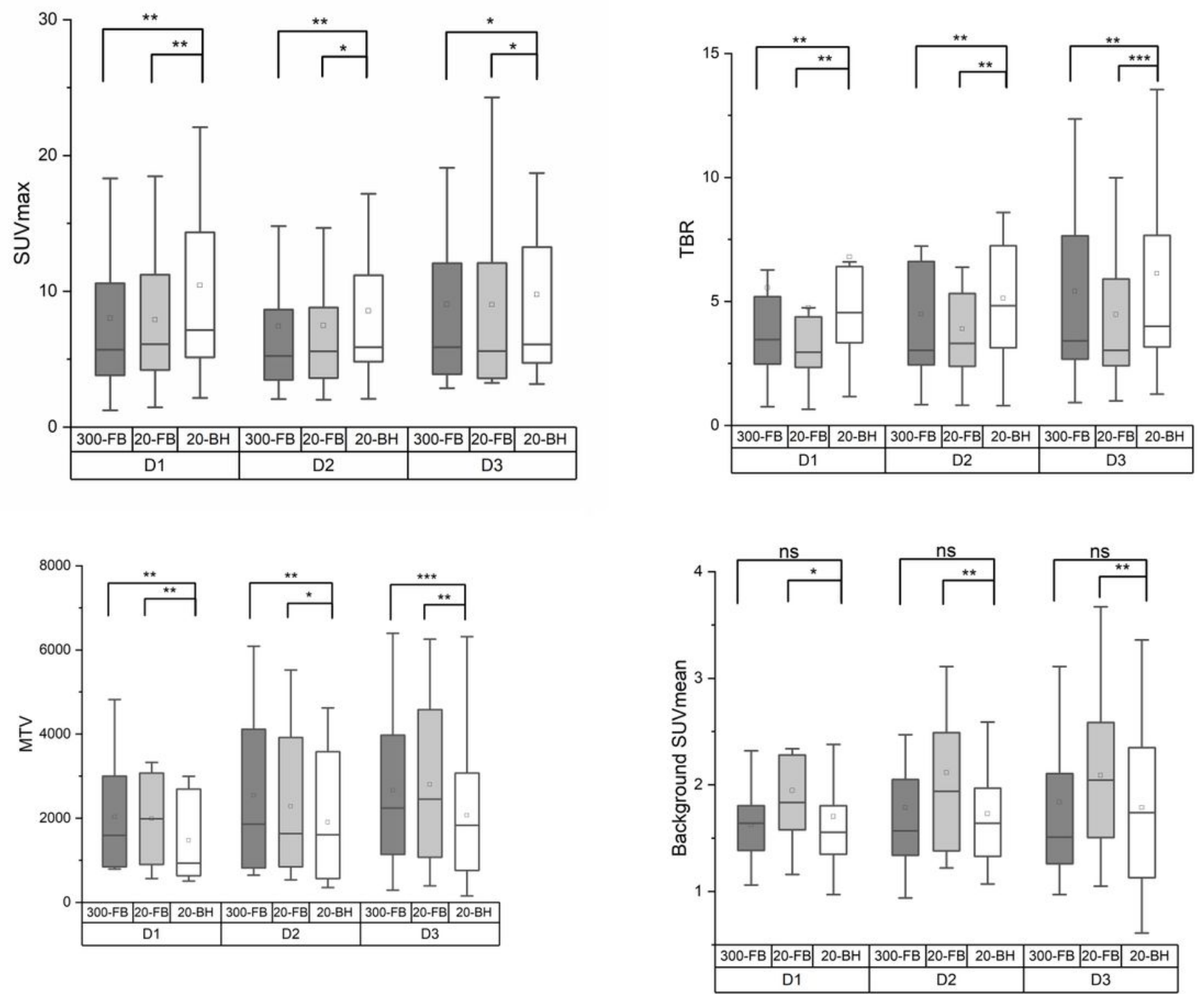

Figure 6

Differences in SUVmax, TBR, MTV and background SUVmean value detected by 300-FB PET (300-FB), 20FB PET (20-FB) and 20-BH PET (20-BH) among D1, D2 and D3 groups. 


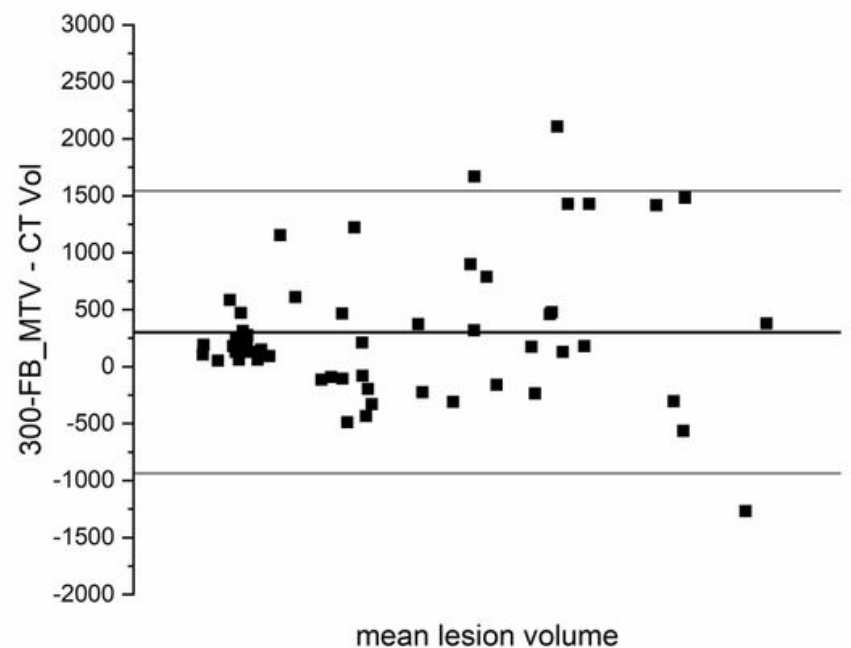

mean lesion volume

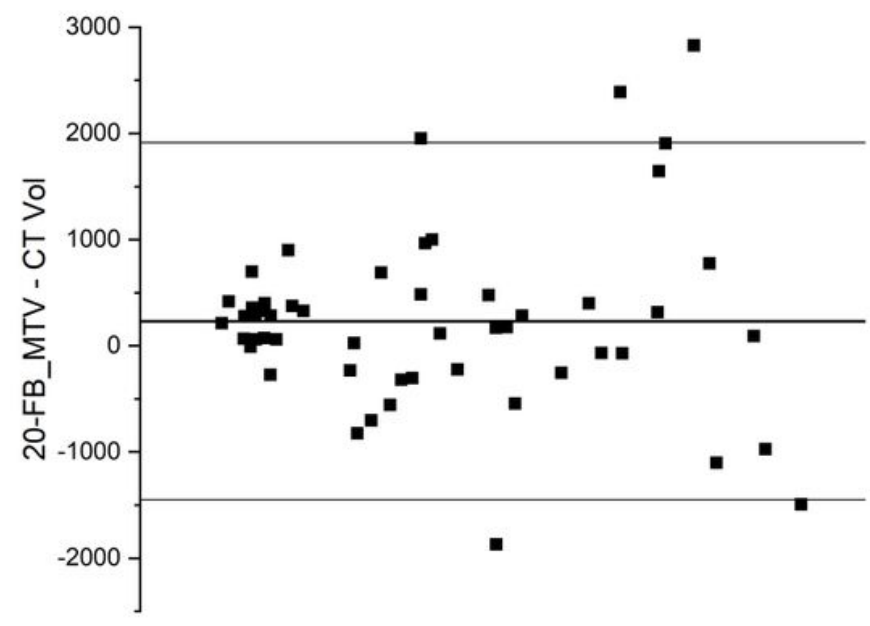

mean lesion volume

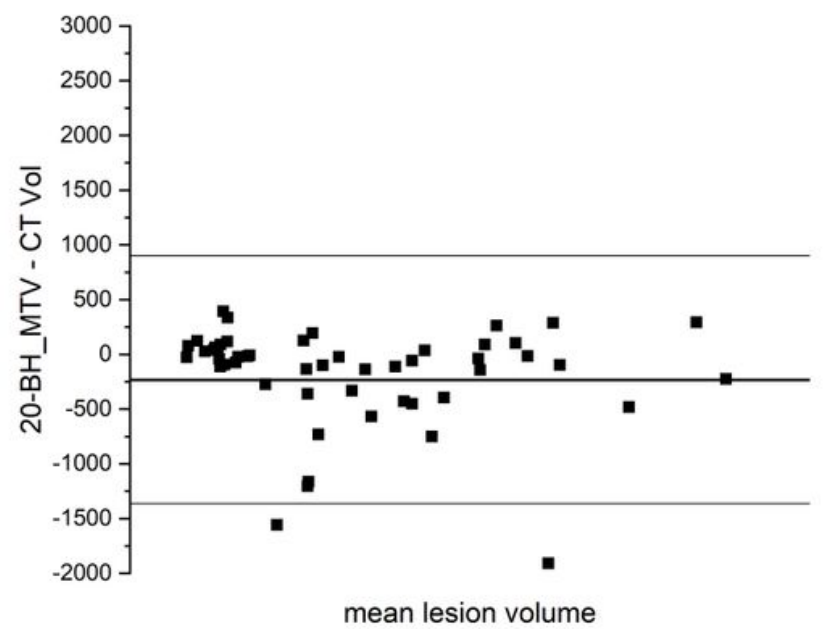

Figure 7

Bland-Altman plots to show agreement between MTV in 300-FB PET (a), 20-FB PET (b) and 20-BH PET (c) acquisitions and lesion volume in BHCT for the 47 lesions. 


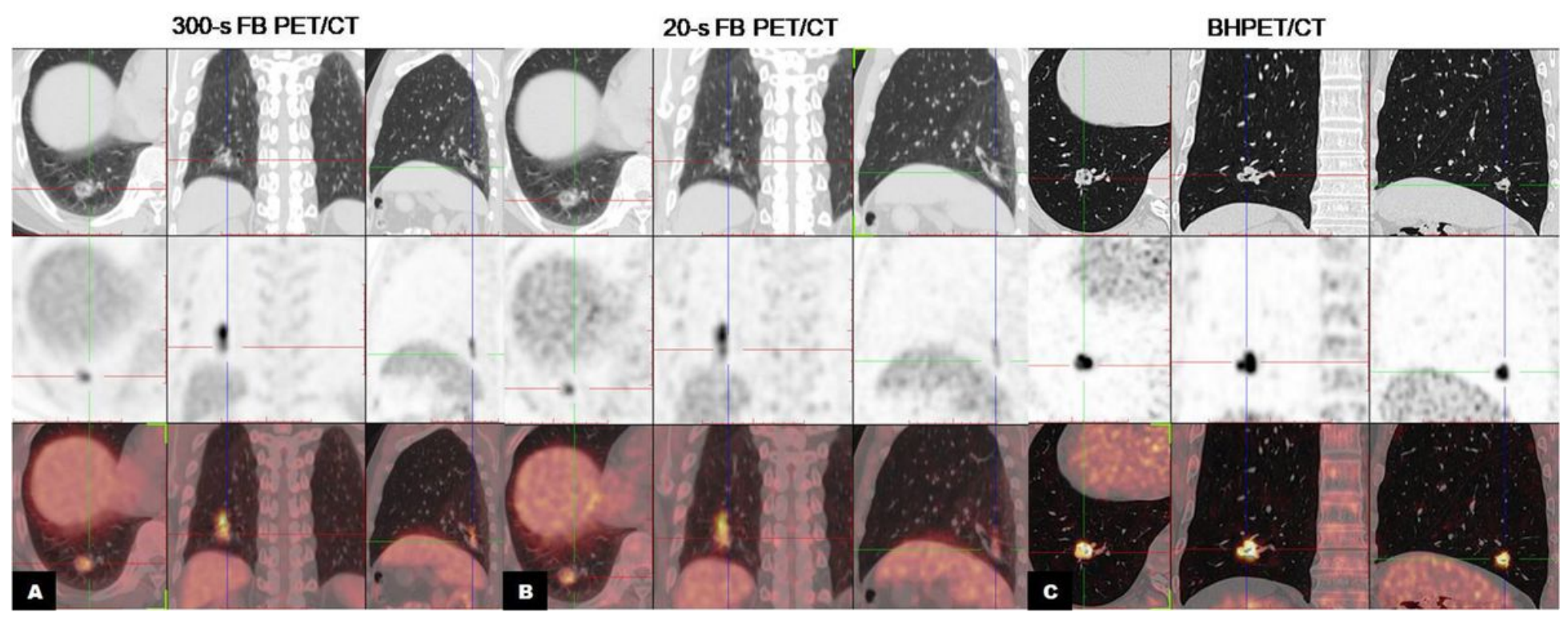

\section{Figure 8}

Axial, coronal, and sagittal chest CT (top row), PET (middle row) and PET/CT (bottom row) images in 59year-old man with solitary solid lung nodule (surgery-proven adenocarcinoma) in right lower lobe. A, 300FB PET/CT; B, 20-FB PET/CT; C, 20-BH PET/CT. Maximum axial diameter of lesion was $16.2 \mathrm{~mm}$. Lesion was not clearly visualized on 300- and 20-FB PET/CT due to respiratory motion blurring but presented significantly clear on 20-BH PET/CT, with increased SUVmax and TBR and accurate lesion volume.

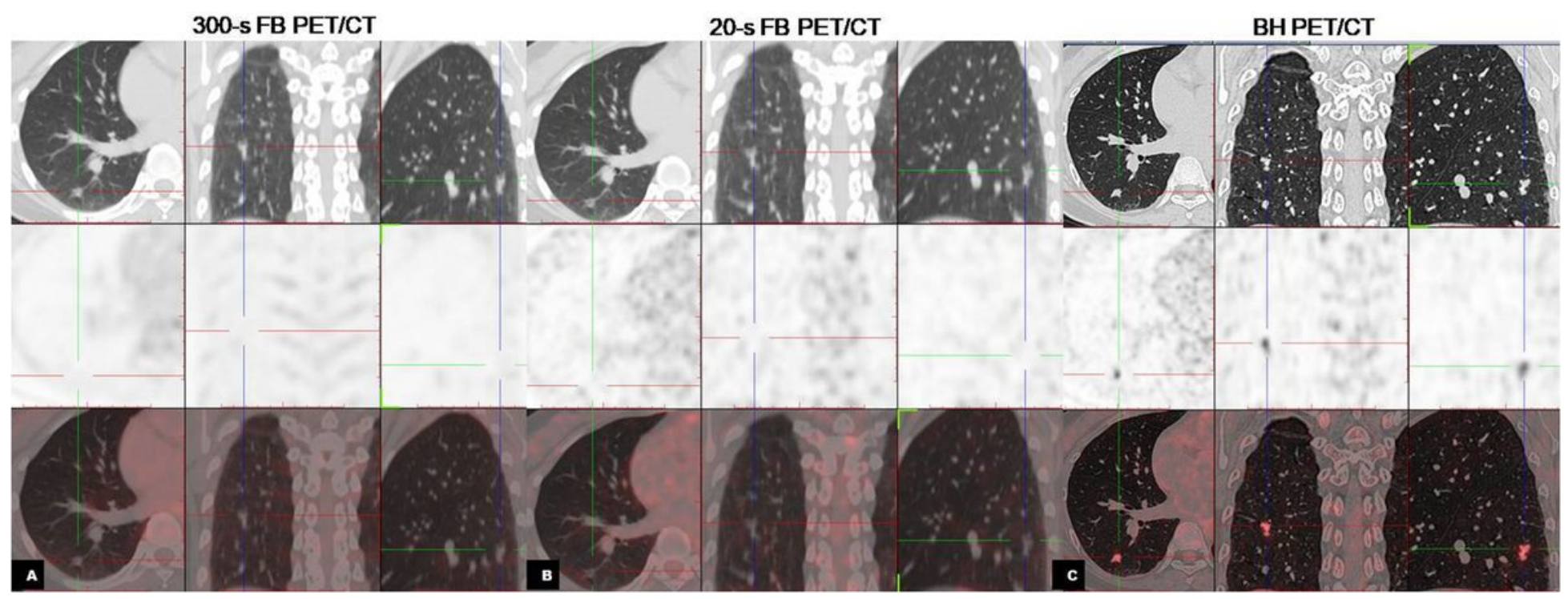

Figure 9

Axial, coronal, and sagittal chest CT (top row), PET (middle row) and PET/CT (bottom row) images in 44year-old woman with solitary solid lung nodule (surgery-proven adenocarcinoma) in right lower lobe. A, 300-s FB PET/CT; B, 20-s FB PET/CT; C, BH PET/CT. Maximum axial diameter of lesion was $9.2 \mathrm{~mm}$. Lesion was FDG negative on 300- and 20-FB PET but FDG positive on 20-BH PET. 


\section{Supplementary Files}

This is a list of supplementary files associated with this preprint. Click to download.

- Tables.pdf 